\title{
Mixed order robust adaptive control for general linear time invariant systems
}

\author{
Javier A. Gallegos ${ }^{\mathrm{a}, *}$, Manuel A. Duarte-Mermoud ${ }^{\mathrm{a}, \mathrm{b}}$, \\ Rafael Castro-Linares ${ }^{c}$ \\ ${ }^{a}$ Department of Electrical Engineering, University of Chile, Av. Tupper 2007, Santiago, Chile \\ ${ }^{\mathrm{b}}$ Advanced Mining Technology Center, University of Chile, Av. Tupper 2007, Santiago, Chile \\ ${ }^{\mathrm{c}}$ Department of Electrical Engineering, CINVESTAV, Av. IPN 2508, México DF, México
}

Received 23 September 2016; received in revised form 2 November 2017; accepted 7 February 2018

Available online 14 March 2018

\begin{abstract}
We provide a solution to the adaptive control problem of an unknown linear system of a given derivation order, using a reference model or desired poles defined in a possibly different derivation order and employing continuous adjustment of parameters ruled by possibly another different derivation order. To this purpose, we present an extension for the fractional settings of the Bezout's lemma and gradient steepest descent adjustment. We analyze both the direct and indirect approaches to adaptive control. We discuss some robustness advantages/disadvantages of the fractional adjustment of parameters in comparison with the integer one and, through simulations, the possibility to define optimal derivation order controllers.
\end{abstract}

(C) 2018 The Franklin Institute. Published by Elsevier Ltd. All rights reserved.

\section{Introduction}

The adaptive control problem can be expressed as given an unknown system to design a control input to drive the system output in a desired way. It is assumed the availability of its output and the handiness to manipulate the input. The simplest strategy is based on

\footnotetext{
* Corresponding author.

E-mail address: jgallego@ing.uchile.cl (J.A. Gallegos).
} 
continuously adjusted parameters which enter linearly in the control function paired with signals generated from the input and output functions [1-4]. Those parameters are the adaptive elements in the control design, i.e., they are varied to minimize the discrepancy with respect to the control objective.

The importance of this problem is reflected in many extensions and applications being constantly developed up to date [5-8]. It is due to the fact that total and precise knowledge of a system at each instant is not possible since real systems are closer to an infinite dimensional ones, whereby there will be missing terms in any finite dimensional modeling and even if one could know the parameters at some instant, they rarely will remain constant along the time.

The recent understanding on fractional order systems gives a wider perspective of the problem. Indeed, the unknown system can be given by some order of derivation; the desired output can be specified by some other order of derivation and the continuous adjustment of parameters can be realized by using another order of derivation. We term this view as mixed order adaptive control. In [9], this strategy was employed in the model reference adaptive control (MRAC) problem. Although no analytic proof was provided there, simulations showed that the speed of convergence, stability and transient behavior depend on the chosen order of derivation for the adjustment of parameters and the chosen order on the reference model. As a consequence, the inclusion of this fractional order perspective in the adaptive problem allows us to enlarge the degree of freedom available to the designer, even though the unknown linear system is just of integer order.

To illustrate the main theoretical unsolved problems in mixed order adaptive control, we cite the recent works $[10,11]$ where it is considered the adaptive pole placement problem. A Diophantine equation appears when the unknown integer order system is to match a fractional order system, involving no commensurate polynomials and hence the Bezout's Lemma is unsuited. An approximation of fractional operator as an integer system is used avoiding this problem. To adjust the parameters, a discretization and a regression model are employed, losing the fractional degree of freedom. In this simplified non-fractional setting, no conditions were posited to assure convergence or even stability. Their simulations again suggest improvement in the presence of disturbances.

The control problem of systems with uncertainties is an active research field in the study of fractional systems. In multi-agent consensus problem [12], the agents are ruled by fractional linear systems subjected to parametric uncertainty. In $[13,14]$, stability and robustness of fractional control of known linear system were studied.

A strong assumption of integer order adaptive control is that the unknown linear system must be of minimal phase. The mixed order indirect approach can take advantage of the fractional partial cancellation of unstable zeros at lower ranges of frequency as proposed in [15]. This can be achieved with an adaptive indirect compensator, generalizing the procedure in [16].

Our main contribution is to give a theoretical solution to the MRAC and pole placement adaptive problems in both direct and indirect approaches using this mixed perspective, for SISO linear systems of arbitrary finite dimension, giving analytic support to the simulation studies in [9-11]. In particular, we provide a generalization of the Bezout's Lemma in [17] for non-commensurate polynomials and a generalization of the fractional adjustment in [16] by adding a perturbation term in the error equation. We show a robustness improvement of the fractional adjustment. 
In comparison with $[18,19]$, which employ a Lyapunov approach, we give convergence and robustness conditions for the error in the adaptive model reference problem and we do it for the mixed order strategy. In [20] a mixed robust strategy using sliding surface was developed, showing improvement in the performance but it is non adaptive and requires the knowledge of the state. An alternative approach to control unknown (interval) fractional system is exposed in [21], using LMI inequalities and in [22] using Minkowski sum. In comparison to our method, it is non mixed, it only resolves the stabilization problem (non tracking), the parameters must lie in some known bounded convex set and the requirements are involved.

Although our results are constrained to linear systems, they can be used in local approximation of nonlinear systems. However, and for special disturbed fractional nonlinear system, non mixed adaptive regularization has been developed based in backstepping technique in [23], which can be particularized to linear systems, though they do not give a proof of the error convergence. On the other hand, many non-linear applied systems admit a description compatible with the error model I in the estimation part (see e.g., [24] for fuzzy techniques), whereby an indirect mixed order controller can be developed, following our approach. It can be useful to combine the projection extension proposed in this paper with the adaptive tracking of non linear system proposed in [25].

The contributions of this paper are structured as follows: In Section 2, we develop the mathematical fundamentals necessary to the adaptive control design. In Sections 3 and 4, we present a solution to the adaptive MRAC and pole placement direct and to the indirect model reference and pole placement adaptive control problems. Section 5 is devoted to analyse the robust behavior improvements of the fractional gradient adjustment of parameters. Section 6 provides simulation examples of the proposed methods and in Section 7 the conclusions are indicated.

\section{Preliminary results}

In this section, general mathematical concepts and propositions are developed to be applied in adaptive problems.

\subsection{Lebesgue spaces}

Lebesgue spaces are defined by

$\mathcal{L}^{p}(S, \mu):=\left\{f: S \rightarrow \mathbb{R} \mid\|f\|_{p} \equiv\left(\int_{S}|f|^{p} \mathrm{~d} \mu\right)^{\frac{1}{p}}<\infty\right\}$,

where $\mu$ is the Lebesgue measure and, in general, $S=\mathbb{R}_{\geq 0}$ [1]. The definition for vector functions is straightforward. For $\alpha \in \mathbb{R}_{\geq 0}$, we define the following sets

$\mathcal{L}_{\alpha}^{p}\left(\mathbb{R}_{\geq 0}\right):=\left\{f:[0, \infty) \rightarrow \mathbb{R} \mid(\forall t>0)\left({ }_{0} I_{t}^{\alpha}|f|^{p}(t)\right)^{\frac{1}{p}}<\infty\right\}$,

where ${ }_{0} I_{t}^{\alpha} f(t):=\frac{1}{\Gamma(\alpha)} \int_{0}^{t}(t-\tau)^{\alpha-1} f(\tau) d \tau$ is the fractional integral of order $\alpha$ [26]. Note that $\left({ }_{0} I_{t}^{\alpha}|f|^{p}(t)\right) \frac{1}{p}, \forall t>0$ does not necessarily imply that $\lim _{t \rightarrow \infty}\left({ }_{0} I_{t}^{\alpha}|f|^{p}(t)\right)^{\frac{1}{p}}<\infty$ when $\alpha<1$, since this limit might not exist [28, Proposition 3], but for $\alpha \geq 1, \lim _{t \rightarrow \infty}\left({ }_{0} I_{t}^{\alpha}|f|^{p}(t)\right)^{\frac{1}{p}}<\infty$ [27].

We mostly use here $\mathcal{L}_{\alpha}^{1}, \mathcal{L}_{\alpha}^{2}$. Since $|f+g| \leq|f|+|g|$ and $(f+g)^{2} \leq 2 f^{2}+2 g^{2}$, it follows that $\mathcal{L}_{\alpha}^{1}, \mathcal{L}_{\alpha}^{2}$ are vector spaces. For any fixed $T>0$, an inner product can be defined by 
$\langle f, g\rangle_{T, \alpha}:={ }_{0} I_{T}^{\alpha}(f g)$ since it is linear, symmetric and definite positive (module function of null measure). With this inner product, $\mathcal{L}_{\alpha}^{1}, \mathcal{L}_{\alpha}^{2}$ have the structure of normed vector spaces. Moreover, Cauchy-Schwarz inequality holds, namely $\left|\langle f, g\rangle_{T, \alpha}\right|^{2} \leq\langle f, f\rangle_{T, \alpha}\langle g, g\rangle_{T, \alpha}$. Thus (omitting $0, T$ in the integral operator for simplicity),

$\left|I^{\alpha} f g\right|^{2} \leq\left(I^{\alpha} f^{2}\right)\left(I^{\alpha} g^{2}\right)$.

and for $g \equiv 1$,

$I^{\alpha}\left(f^{2}\right)(T) \geq \frac{1}{T^{\alpha}}\left|I^{\alpha}(f)(T)\right|^{2}$.

Therefore, $\mathcal{L}_{\alpha}^{2}([0, T]) \subseteq \mathcal{L}_{\alpha}^{1}([0, T])$ for any $T>0$.

Remark 1. If $f$ is such that $f \in \mathcal{L}^{p}$ then $f \in \mathcal{L}_{\alpha}^{p}$ since otherwise $I|f|^{p}=I^{1-\alpha} I^{\alpha}|f|^{p}$ diverges. More generally, if $f \in \mathcal{L}_{\beta}^{p}$ then $f \in \mathcal{L}_{\alpha}^{p}$ provided that $\alpha \leq \beta$.

\subsection{Error Model I}

It will be shown in Sections 3 and 4, that adaptive control problems can be formulated using the following fractional version of the gradient adjustment law for the Error Model I [1], that is

$$
\left\{\begin{array}{l}
e=\phi^{T} w+f \\
D^{\alpha} \phi=-\gamma e w
\end{array}\right.
$$

where $\gamma>0$ is a constant arbitrarily chosen, the first equation of (4) is called Error Model I, $e$ : $[0, \infty) \rightarrow \mathbb{R}$ is the error function, $w:[0, \infty) \rightarrow \mathbb{R}^{n}$ is the information signal, $\phi:[0, \infty) \rightarrow$ $\mathbb{R}^{n}$ is the parametric error and $f:[0, \infty) \rightarrow \mathbb{R}$ is an additional function which takes account of initial conditions, perturbation terms and/or unmodeled dynamics. Without loss of generality, it is assumed that $w$ is bounded since it can be normalized by using $\gamma\left(\right.$ e.g. $\gamma(t):=\frac{\gamma^{\prime}}{1+w^{T}(t) w(t)}$ where the constant is now $\gamma^{\prime}$ ).

We recall the definition of the set of persistently exciting functions $P E(n)$, namely the set of bounded functions such that [3],

$P E(n):=\left\{w:[0, \infty) \rightarrow \mathbb{R}^{n} \mid\left(\exists \epsilon, T_{0}>0\right)\right.$ st. $\left.(\forall t>0) \int_{t}^{t+T_{0}} w(\tau) w^{T}(\tau) d \tau \geq \epsilon I_{n}\right\}$.

The following theorem generalizes [16, Theorem 2 and Proposition 1] where $f \equiv 0$ was assumed. Without loss of generality, we assume $\gamma=1$ (although this constant can improve the speed of convergence [16], we choose a simplified version for sake of clarity).

Theorem 1. Consider system (4) with Caputo derivative for $0<\alpha \leq 1$. Let $f$ be bounded, continuously differentiable and uniformly continuous function such that $I^{\alpha} f^{2}$ is bounded by a constant number $C$. Then,

(i) $I^{\alpha} e^{2}$, $e$ and $\phi$ are bounded functions.

(ii) If $w$ is, in addition, uniformly continuous and f converges to zero then $\liminf _{t \rightarrow \infty} e^{2}(t)=$ 0 and, for any $\epsilon>0,|e|>\epsilon$ only can occur at time intervals of finite length, with such intervals necessarily occurring with an unbounded increasing separation. Moreover, the $R M S$ value of e converges to zero.

(iii) If $f$ converges to zero, $w \in P E(n)$ then $\phi$ and e converge to zero. 
(iv) Let $g:[0, \infty) \rightarrow \mathbb{R}^{n}$ be bounded, continuously differentiable and uniformly continuous functions such that $I^{\alpha}\|g\|^{2}$ is bounded. If $v \in P E(n)$ then $w:=v+g \in P E(n)$ and therefore, by replacing this $w$ in Eq. (4), $\phi$ and e converge to zero.

\section{Proof.}

(i) Define $V(t):=\phi^{T} \phi(t)+C-1 / 2 I^{\alpha} f^{2}(t)$. By [29, Lemma 1]([36, Lemma 1]), we have that $D^{\alpha}\left[\phi^{T} \phi\right](t) \leq 2 \phi^{T} D^{\alpha} \phi(t)$, where the differentiability condition on $w$ and $f$ guarantees its application [31, Property 12]. Hence, $D^{\alpha} V \leq-2\left(\phi^{T} w\right)^{2}-2 \phi^{T} w f-1 / 2 f^{2}$. By completing the square,

$D^{\alpha} V \leq-2\left(\phi^{T} w+1 / 2 f\right)^{2} \leq 0$.

By $\alpha$-integration of this expression, we obtain $V(t) \leq V(0),\left(\phi^{T} w+f / 2\right) \in \mathcal{L}_{\alpha}^{2}$ and then $\phi$ is bounded. Since $f \in \mathcal{L}_{\alpha}^{2}$, it follows that $e=\left(\phi^{T} w+f\right) \in \mathcal{L}_{\alpha}^{2}$. Since $e=\phi^{T} w+f$ and $f, w$ are bounded by hypothesis, $e$ is also bounded.

(ii) From uniform continuity of $w, \phi$ (since $D^{\alpha} \phi=-w w^{T} \phi-w f$ is bounded [31, Proposition 1]), $f$ (since it is continuous and converges to zero) and from boundedness of $e$, we conclude uniform continuity of $e^{2}$. Then, for every $\epsilon>0, e^{2}(t)>\epsilon$ only occurs at time intervals of finite length. Such intervals necessarily occur with an increasing separation of time, otherwise $I^{\alpha} e^{2}$ diverges, since there would exist a finite time $T$ large enough where always occurs one of those intervals in the intervals $[i T,(i+1) T]$ for any $i \in \mathbb{N}$, whereby, using [31, Example 5], $I^{\alpha} e^{2}$ diverges. Note that

$t^{-1} \int_{0}^{t} e^{2}(\tau) d \tau=t^{-1} I^{1-\alpha}\left[I^{\alpha} e^{2}\right] \leq C^{\prime} t^{-1} t^{1-\alpha} \rightarrow 0$

when $t \rightarrow \infty$, where $C^{\prime}$ is the bound on $I^{\alpha} e^{2}$. Hence

$$
\lim _{t \rightarrow \infty} \sqrt{\frac{1}{t} \int_{0}^{t} e^{2}(\tau) d \tau}=0 .
$$

(iii) System (4) can be written as

$D^{\alpha} \phi=-w w^{T} \phi-w f$,

where $w f$ converges to zero since $w$ is bounded. The claims follows from [35, Theorem 2.1] together with [35, Section 3.1].

(iv) By replacing $w=v+g$ in

$$
\left|\frac{1}{T} \int_{s}^{s+T}\left[w(t) w(t+\tau)^{T}-v(t) v(t+\tau)^{T}\right] d t\right|
$$

we obtain

$$
=\left|\frac{1}{T} \int_{s}^{s+T}\left[g(t) v(t+\tau)^{T}+v(t) g(t+\tau)^{T}-g(t) g(t+\tau)\right] d t\right| .
$$

Using Cauchy-Swchartz by and evaluating at $\tau=0$, we have

$$
\leq\left[\frac{1}{T} \int_{s}^{s+T}\|g(t)\|^{2} d t\right]^{1 / 2}\left[\frac{1}{T} \int_{s}^{s+T}\|v(t)\|^{2} d t\right]^{1 / 2}
$$




$$
\begin{aligned}
& +\left[\frac{1}{T} \int_{s}^{s+T}\|g(t)\|^{2} d t\right]^{1 / 2}\left[\frac{1}{T} \int_{s}^{s+T}\|v(t)\|^{2} d t\right]^{1 / 2} \\
& +\frac{1}{T} \int_{s}^{s+T}\|g(t)\|^{2} d t
\end{aligned}
$$

Since $\quad v \in P E(n)$, we have $\lim _{T \rightarrow \infty}\left[\frac{1}{T} \int_{s}^{s+T}\|v(t)\|^{2} d t\right]^{1 / 2}=\operatorname{trace}\left(R_{v}(0)\right) \quad$ where $R_{v}(0)>0$ is the auto covariance of $v$ [1, Proposition 2.7.1]. Using a similar argument of the proof of the last statement of part (ii) $\lim _{T \rightarrow \infty}\left[\frac{1}{T} \int_{s}^{s+T}\|g(t)\|^{2} d t\right]^{1 / 2}=0$. Hence,

$$
\lim _{T \rightarrow \infty}\left|\frac{1}{T} \int_{s}^{s+T}\left[w(t) w(t+\tau)^{T} d t-v(t) v(t+\tau)^{T}\right] d t\right|=0
$$

where by

$$
\lim _{T \rightarrow \infty}\left|\frac{1}{T} \int_{s}^{s+T} w(t) w(t+\tau)^{T} d t\right|=R_{\nu}(0)>0
$$

implying $w \in P E(n)$ by [1, Proposition 2.7.1]. The last claims follows from part (iii).

Remark 2. (i) This result allows to neglect $\mathcal{L}_{\alpha}^{2}$ additive terms in adaptive problems involving Eq. (4). By Remark 1, $\mathcal{L}^{2}$ terms can be in particular dropped.

(ii) In Theorem 1(i), the boundedness of $w$ is used to prove the boundedness of $e$. This hypothesis can be replaced by requiring uniform continuity of the variables involved, since in this case $e$ only could diverge at infinite time, but this contradicts that $e \in \mathcal{L}_{\alpha}^{2}$; and hence, $e$ will be bounded.

The following result provides mild conditions for convergence to zero of the error function $e$ of system (4) without requiring convergence of $\phi$ to zero. It is needed that some of the components of $w$ form a $P E$ function instead of the all vector. Therefore, the case $n=1$ is not modified and the case $n=2$, it is enough a senusoidal function.

Proposition 1. Consider system (4) for $n \geq 3$. Let $w:[0, \infty) \rightarrow \mathbb{R}^{n}$ be a bounded, continuously differentiable and uniformly continuous function.

(i) If $\alpha=1$ then e converges to zero.

(ii) Let $0<\alpha \leq 1$ for Caputo derivative. If some of the components of $w$ form a vector in $P E(k)$ for $k \geq 1$ and the other are linearly dependent of theses, then e converges to zero.

\section{Proof.}

(i) See for instance [1, Theorem 2.4.5].

(ii) Without loss of generality, assume that $w$ has its first $k$ components linearly independent forming a $P E(k)$ function and the rest $(n-k)$ linearly dependent of the first $k$ components. We will prove that there exists a constant orthogonal matrix $A$ such that the vector $A w$ has its first $k$ components identical to $w$ and zero otherwise. For this, the $n^{2}$ elements of $A$ must satisfies that $\sum_{j=1}^{n} a_{i j} w_{j}=w_{i}$ for $i \leq k, \sum_{j=1}^{n} a_{i j} w_{j}=0$ otherwise, and $A^{T} A=I$. The first equations has solution (for instance, $a_{i j}=1$ if $i=j$ and 0 otherwise). The second equations can be solved because of the linearly dependent conditions but to assure independence of 
the $(n-k)$ equation, we quit elements in the following way: in equation $i=k+j$ we quit the element $w_{k+j}$. The last equation gives, by symmetry, $n(n+1) / 2$ linearly independent equations. Hence, there are $n(n+1) / 2+n$ l.i. equations. The system of equations has solution provided that $n(n+1) / 2+n \leq n^{2}$ i.e., $3 \leq n$.

The adjustment law is equivalent to $D^{\alpha} \phi=-w w^{T} \phi$. Using the transformation, $\hat{\phi}:=A \phi$, we obtain $D^{\alpha} \hat{\phi}=-A w w^{T} A^{T} \hat{\phi}=-A w(A w)^{T} \hat{\phi}$. By Theorem 1(iii), the first $k$ components of $\hat{\phi}$ converge to zero. The remaining $(n-k)$ components are constant but the corresponding components in $A w$ are null. Thus, $(A w)^{T} \hat{\phi}$ converges to zero. But, $(A w)^{T} \hat{\phi}=$ $(A w)^{T} A \phi=w^{T} A^{T} A \phi=w^{T} \phi=e$, whereby $e$ converges to zero.

Remark 3. The asymmetry in the conditions for convergence of $e$ is ultimately related to the more restricted generalization of Barbalat's Lemma for $0<\alpha<1$, since for uniformly continuous function there is a counterexample [27, Proposition 4] of non convergence when $0<\alpha<1$ but convergence for $\alpha=1$.

Example 1. By using Proposition 1, if $w$ is a non null constant vector, then $\lim _{t \rightarrow \infty} e(t)=0$, since $w$ has a component $P E(1)$ and all the other are linearly dependent of it. This fact can be proved for any $0<\alpha<2$. In fact, $D^{\alpha} w^{T} \phi=-w^{T} w w^{T} \phi$. Thereby, $D^{\alpha} e=-w^{T} w e=-\|w\|^{2} e$ which implies $\lim _{t \rightarrow \infty} e(t)=0$. This can explain, for the case of $w$ constant, the speed improvement for cases of $\alpha>1$ in comparison with $\alpha=1$ obtained in [9] by simulation, since the solution in the fractional case converges faster to zero.

More importantly, for any $0<\alpha \leq 1$, if $\lim _{t \rightarrow \infty} w(t)=w_{0}$ with $w_{0}$ a constant non null vector, then $D^{\alpha} w_{0}^{T} \phi=-w_{0}^{T} w w^{T} \phi=-w_{0}^{T}\left(w_{0}+f\right)\left(w_{0}+f\right)^{T} \phi$ with $\lim _{t \rightarrow \infty} f(t)=0$. Therefore, $D^{\alpha} w_{0}^{T} \phi=-w_{0}^{T} w_{0} w_{0}^{T} \phi+w_{0}^{T}\left(f w_{0}^{T}+w_{0} f^{T}+f f^{T}\right) \phi$. Since, $\phi$ is bounded by Theorem 1 , the second term in the right hand side vanishes. By using the above paragraph and [31, Theorem 5], we conclude that $\lim _{t \rightarrow \infty} w_{0}^{T} \phi(t)=0$, whereby $\lim _{t \rightarrow \infty} e(t)=\lim _{t \rightarrow \infty}\left(w_{0}^{T}+\right.$ $f(t)) \phi(t)=0$.

In particular, if in the Laplace domain $w=H(s) r$ where $H$ is a vector transfer function asymptotically stable and $r$ is a constant function, we conclude that $\lim _{t \rightarrow \infty} e(t)=0$.

\subsection{Gradient method with projection}

Besides of Eq. (4), it will be required in Section 3 and Section 5 a generalization of the gradient algorithm with projection expressed in the integer order case by [1, Theorem 2.4.3],

$\frac{d}{d t} \theta(t)=\left\{\begin{array}{l}-e(t) w(t) \text { if }\left(\theta(t) \in \operatorname{int}(C) \text { or } e w^{T} N>0\right) \\ \operatorname{Pr}(-e(t) w(t)) \text { if }\left(\theta(t) \in \partial C \text { and } e w^{T} N \leq 0\right)\end{array}\right.$

where $\operatorname{int}(C)$ is the interior of some bounded set $C, \partial C$ is the boundary of $C, \operatorname{Pr}(z)$ is the projection of $z$ into the hyperplane tangent to $\partial C$ at $\theta$ and $N$ is the outward normal to the hyperplane tangent to $\partial C$ at $\theta(t)$. For simplicity, we use $e=\phi^{T} w$ with $\phi:=\theta-\theta^{*}$ where $\theta^{*} \int(C)$

If $\hat{\theta}(0) \in C$ then $\hat{\theta}(t) \in C$ for all $t>0$ and all properties of gradient scheme are preserved, since by construction $\dot{\hat{\theta}}^{T} N \leq 0$ hence $\hat{\theta}(t)$ is trapped in $C$. There is efficient computational implementations of this law provided that $C$ has simple structure (e.g., a ball).

Let consider $0<\alpha<1$, a fixed but arbitrary $\epsilon \geq 0$ and the adaptive law

$$
D^{\alpha} \theta=\left\{\begin{array}{l}
-e w \text { if }\left(\theta \in \operatorname{int}(C) \text { or } e w^{T} N>0\right) \\
\operatorname{Pr}(-e w)-\epsilon N \text { if }\left(\theta \in \partial C \text { and } e w^{T} N \leq 0\right)
\end{array}\right.
$$


We will prove that (a) the properties are preserved with respect to the unmodified law (system (4)) and that (b) $\theta \in C$. Indeed,

(a) The modification with respect to the unmodified law occurs when $\theta \in \partial C$ and $e w^{T} N \leq 0$. In that case, by decomposition, $-e w=T+\beta N$ where $T$ is the tangent to the hyperplane tangent to $\partial C$ at $\theta$. Since $e w^{T} N \leq 0$ we have that $\beta>0$. Since $\theta \in \partial C$ and $\theta^{*} \in \operatorname{int}(C)$ we have $\left(\theta-\theta^{*}\right)^{T} N=\phi^{T} N>0$. It follows that, $2 D^{\alpha} \phi^{T} \phi \leq \phi^{T}(-e w-(\beta+\epsilon) N) \leq-e \phi^{T} w=-e^{2}$ (using [29, Lemma 1] for the first inequality). The case when $f \neq 0$ is similar by employing the proof of Theorem 1(i).

(b) We will show that $\theta$ cannot escape. When $\theta \in \partial C, D^{\alpha} \theta(t)=T+\beta N$ and $\beta>0$. We note that $D^{\alpha} \theta$ is forced to be $D^{\alpha} \theta\left(t^{+}\right)=T-\epsilon N$ with $\epsilon>0$ by the adaptive law. Since $D^{\alpha} \theta\left(t^{+}\right)=I^{1-\alpha} \dot{\theta}\left(t^{+}\right)=\frac{1}{\Gamma(\alpha)} \int_{0}^{t}\left(t^{+}-\tau\right)^{\alpha-1} \dot{\theta}(\tau) d \tau+\frac{1}{\Gamma(\alpha)} \int_{t}^{t^{+}}\left(t^{+}-\tau\right)^{\alpha-1} \dot{\theta}(\tau) d \tau$, the first term being $T+\beta N$. Hence, $\dot{\theta}\left(t^{+}\right)$must follow the $-N$ direction, otherwise $I^{1-\alpha} \dot{\theta}\left(t^{+}\right)$ cannot follow the $-N$ direction. Therefore $\theta$ remains in C. Since $\theta(0) \in C, \theta(t) \in C$ for all $t>0$.

Remark 4. For part (b), it is not necessary the specific form of the error equation. It has application when the error function has an extra additive noise term (see Section 5).

\subsection{Boundedness under perturbations}

We give a result which will be used in Section 4 to assure boundedness of the solutions under parametric variations (due to adaptive adjustment in Section 4) and noise perturbations, generalizing the result for adaptive integer system in [3, Lemma 2.1].

Theorem 2. Consider the following Caputo system

$D^{\alpha} x(t)=(A+\delta A(t)) x(t)+v(t)$,

where $\alpha \in(0,1], x:[0, \infty) \rightarrow \mathbb{R}^{n}$ and $v:[0, \infty) \rightarrow \mathbb{R}^{n}$ is a bounded continuous function $\left(\|v\|_{\infty}<v_{0}\right)$. Let $x_{0} \in \mathbb{R}^{n}$ be the arbitrary initial condition. Suppose that $\delta A:[0, \infty) \rightarrow \mathbb{R}^{n \times n}$ is a continuous matrix function such that $\lim _{t \rightarrow \infty} \delta A(t)=0$. Assume that $A \in \mathbb{R}^{n \times n}$ is a constant matrix with its spectrum satisfying $\sigma(A) \subset\left\{\lambda \in \mathbb{C}-\{0\}:|\arg (\lambda)|>\frac{\alpha \pi}{2}\right\}$. Then $x$ is a bounded function.

Proof. Define the operator in the space of continuous functions $\mathcal{C}([0, \infty))$ by

$\mathcal{T}_{x_{0}}(\xi)(t):=E_{\alpha}\left(t^{\alpha} A\right) x_{0}+\int_{0}^{t} \tau^{\alpha-1} E_{\alpha, \alpha}(A, \tau)[\delta A \xi](t-\tau) d \tau+\int_{0}^{t} \tau^{\alpha-1} E_{\alpha, \alpha}(A, \tau) \nu(t-\tau) d \tau$

where $E_{\alpha}, E_{\alpha, \alpha}$ are Mitteg-Laffler functions [26]. Let $\epsilon>0$ be a real number. Since $\delta A$ is continuous and converges to zero, it is bounded. Then, by taking $\xi(t) \in B_{\mathcal{C}_{\infty}}(0, \epsilon):=\{\xi \in$ $\left.\mathcal{C}\left([0, \infty) ; \mathbb{R}^{n}\right):\|\xi\|_{\infty} \leq \epsilon\right\}$ and using that $\int_{0}^{t} \tau^{\alpha-1}\left\|E_{\alpha, \alpha}\left(\tau^{\alpha} A\right)\right\| d \tau<\infty$ ([34, Theorem 3]), we have

$\left\|\mathcal{T}_{x_{0}}(\xi)(t)\right\|_{\infty} \leq C\left(\epsilon,\left\|x_{0}\right\|, v_{0}\right)<\infty$

where $C\left(\epsilon,\left\|x_{0}\right\|, v_{0}\right)$ is a constant term depending on the bound $v_{0}, \epsilon,\left\|x_{0}\right\|$ (the constrain on $\sigma(A)$ implies that $E_{\alpha}\left(t^{\alpha} A\right) x_{0}$ converges to zero).

Since $\|\delta A\|$ converges to zero, we can assume $q:=\left\|\int_{0}^{t} \tau^{\alpha-1} E_{\alpha, \alpha}(A, \tau)[\delta A(t-\tau)] d \tau\right\|<1$, without loss of generality, since we can define a norm $\|\cdot\|_{\beta}$ which is equivalent to $\|\cdot\|_{\infty}$, by 
dividing it by $\sup _{t}\left\|\int_{0}^{t} \tau^{\alpha-1} E_{\alpha, \alpha}(A, \tau)[\delta A(t-\tau)] d \tau\right\|$. Since the terms associated to $v$ are canceled in subtracting the equations, we obtain

$$
\left\|\mathcal{T}_{x_{0}}(\xi)(t)-\mathcal{T}_{x_{0}}(\tilde{\xi})(t)\right\|_{\infty} \leq q\|\xi-\tilde{\xi}\|_{\infty}
$$

Therefore, $\mathcal{T}_{x_{0}}$ restricted to $\mathcal{C}_{\infty}:=\left\{\xi \in \mathcal{C}\left([0, \infty) ; \mathbb{R}^{n}\right):\|\xi\|_{\infty}<\infty\right\}$ is a contraction auto map in a Banach space, whereby it has a fixed point. Since the unique continuous solution to $D^{\alpha} x=(A+Q(t)) x+v$ is written as $x=E_{\alpha}\left(t^{\alpha} A\right) x_{0}+\int_{0}^{t} \tau^{\alpha-1} E_{\alpha, \alpha}(A, t)[\delta A \xi](t-\tau) d \tau+$ $\int_{0}^{t} \tau^{\alpha-1} E_{\alpha, \alpha}(A, t) v(t-\tau) d \tau$, we conclude that $x=\xi$ and hence, $x$ is bounded.

\subsection{Diophantine equation}

In adaptive control it appears the following algebraic problem [2,10]. Given polynomials $A, B, C$, to find $X, Y$ such that the Diophantine equation

$A X+B Y=C$

holds, where polynomials $A, B, C$ could be in general non commensurate. For instance, if $C=$ $s^{\alpha}+1, A=s+1$ and $B=1$, then $X=s^{\beta}, Y=s^{\alpha}+1-s^{\beta+1}-s$ is a solution. Moreover, for any $X$, the pair $(X, Y=(C-A X) / B)$ is a valid solution provided that $B \neq 0$. But we want solutions in the ring of polynomials of non negative real exponents and of real coefficients, denoted in the following as $\mathfrak{P}$ (that it is a ring follows from the ring structure of the real number). Note that $1 / B$ could be written as $\sum b_{i} s^{-\beta_{i}}$ and it would imply an integrator in adaptive control. Although this can be useful, we restrict our analyses to positive exponents since division algorithm could never end with negative exponents.

There are some cases of Eq. (10) that can be solved by inspection, for instance

(i) If $A X+Y=C$ then $Y=C-A X$ for any polynomial $X \in \mathfrak{P}$.

(ii) If $A X+B Y=C$ such that $C=B C^{\prime}$ then $X=B X_{0}$ and $Y=C^{\prime}-A X_{0}$ for any polynomial $X_{0} \in \mathfrak{P}$ (this type of equation will appear in Section 3 ).

However, in Section 3, it will also appear the equation $A X+B Y=1$. In the integer case, Bezout's Lemma ([17, Proposition 1.3.1]) deal with this equation. Note that the more general equation $A X+B Y=C$ has a solution if the equation $A X_{0}+B Y_{0}=1$ has a solution since we can express the former as $X=C X_{0}, Y=C Y_{0}$.

Lemma 1. Consider Eq. (10) with $C$ the greatest common divisor of $A$ and $B$.

(i) If $A, B, C \in \mathfrak{P}$ are commensurate polynomials of the same order, then there exist $X, Y \in \mathfrak{P}$ commensurate polynomials of the same order such that Eq. (10) holds satisfying $\operatorname{deg}(X)<\operatorname{deg}(B)$ and $\operatorname{deg}(Y)<\operatorname{deg}(A)$.

(ii) Let $\mathfrak{P}_{\mathbb{Q}}$ be the set of commensurate and non commensurate polynomials of rational orders with real coefficients. Then $\mathfrak{P}_{\mathbb{Q}}$ is a sub-ring of $\mathfrak{P}$ and if $A, B, C \in \mathfrak{P}_{\mathbb{Q}}$, then there exist $X, Y \in \mathfrak{P}_{\mathbb{Q}}$ such that Eq. (10) holds with $\operatorname{deg}(X)<\operatorname{deg}(B)$ and $\operatorname{deg}(Y)<\operatorname{deg}(A)$.

Proof. (i) Let $\alpha \in \mathbb{R}$ be a common order, i.e., $A(s)=\sum_{i=1}^{n} a_{i} s^{i \alpha}, B(s)=\sum_{i=1}^{m} b_{i} s^{i \alpha}, C(s)=$ $\sum_{i=1}^{l} c_{i} s^{i \alpha}$. By calling $s^{\alpha}=u$, they are rewritten as $A(u)=\sum_{i=1}^{n} a_{i} u^{i}, B(u)=\sum_{i=1}^{m} b_{i} u^{i}$, $C(u)=\sum_{i=1}^{l} c_{i} u^{i}$. By applying Bezout's Lemma to the polynomials $A(u), B(u), C(u)$ where $C(u)$ is the greatest common divisor of $A(u)$ and $B(u)$, we have that there exist polynomials $X(u), Y(u)$ such that $A(u) X(u)+B(u) Y(u)=C(u)$ with $\operatorname{deg}(X)<\operatorname{deg}(B)$ and $\operatorname{deg}(Y)<\operatorname{deg}(A)$. The claim follows by replacing $u=s^{\alpha}$. 
(ii) Consider $A(s)=\sum_{i=1}^{n} a_{i} s^{p_{i} / q_{i}}$. Let $q_{A}$ be the minimal common multiple of $q_{1}, \ldots, q_{n}$ and define $q_{i} r_{i}=q_{A}$ (where $q_{A}, r_{1}, \ldots, r_{n} \in \mathbb{N}$ ). Then $A(s)=\sum_{i=1}^{n} a_{i} s^{p_{i} r_{i}\left(1 / q_{A}\right)}$, whereby $A(s)$ is commensurate in $1 / q_{A}$. Similarly, $B$ and $C$ are commensurate in $1 / q_{B}$ and $1 / q_{C}$, respectively.

Let $q$ be the minimal common multiple of $q_{A}, q_{B}, q_{C}$. Then polynomials $A, B, C$ are commensurate in $1 / q$. In fact, $A(s)=\sum_{i=1}^{n} a_{i} s^{p_{i} r_{i} / q_{A}}=\sum_{i=1}^{n} a_{i} s^{p_{i} r_{i} r_{A}(1 / q)}$ where $q_{A} r_{A}=q\left(r_{A} \in\right.$ $\mathbb{N})$ and $\left(p_{i} r_{i} r_{A}\right) \in \mathbb{N}$ for any $i \in \mathbb{N}$. Note that the dimension of the resultant commensurate polynomial is not $n$ but $\max _{i}\left\{p_{i} r_{i}\right\} r_{A}(1 / q)$. Similarly, for $B$ and $C$. By using an argument similar to the part (i), we conclude that the solutions exist and belong to $\mathfrak{P}_{\mathbb{Q}}$.

Since the exponents belongs to $\mathbb{Q}$, it follows that $\mathfrak{P}_{\mathbb{Q}} \subset \mathfrak{P}$ is a ring, which completes the proof.

Remark 5. (i) As an special case, $A X+B Y=1$ requires that $A, B$ to be coprimes to have solution. On the other hand, some equations can be more efficiently solved by direct inspection. For instance, $p\left(s^{\alpha}-1\right)+q\left(s+s^{1-\alpha}\right)=1$ has as a solution $p=s^{1-\alpha}, q=1$, when $\alpha \leq 1$.

(ii) Since $\mathbb{Q}$ is dense in $\mathbb{R}$ and computation only works with rational numbers, the restriction of rational has no a practical effects.

(iii) Note from the proof that the common commensurate order for arbitrary polynomials in $\mathfrak{P}_{\mathbb{Q}}$ is always $\leq 1$.

Eq. (10) appears also in the following problem (Laplace inversion of transfer functions): to find polynomials $X, Y$ such that $X / A+Y / B=C /(A B)$. Another application is given in the next result.

Corollary 1. Any polynomial in $\mathfrak{P}_{\mathbb{Q}}$ is commensurate in a rational order. In particular, any polynomial in $\mathfrak{P}_{\mathbb{Q}}$ has finite roots in the first Riemann sheet.

Proof. From the proof of Lemma 1, $\sum_{i=1}^{n} a_{i} s^{p_{i} / q_{i}}$ can be written as $\sum_{i=1}^{n} a_{i} s^{p_{i} r_{i} r_{A}(1 / q)}=$ $\sum_{i=1}^{n} a_{i} u^{p_{i} r_{i} r_{A}}$, with $u=s^{1 / q}$. Rewriting it further as $\sum_{i=1}^{m} a_{i}^{\prime} u^{i}=\Pi_{i=1}^{m}\left(u-\gamma_{i}\right)=\Pi_{i=1}^{m}\left(s^{1 / q}-\right.$ $\left.\gamma_{i}\right)$, the claim follows.

\section{Direct adaptive control}

In this approach, the adjustment is done on control parameters to minimize the control objective. Our method consists in formulating the adaptive problem in the framework of an Error Model of Type I (4) to apply a fractional adjustment parameters using Theorem 1. To get such a formulation, we rest in known methodologies for the integer case which must be generalized to take account fractional systems. A matching equation for non commensurate polynomials is thus obtained, where our considerations on Diophantine equations will be applied.

In the Narendra-Valavani approach [2, Chapter 5] the output error turns out to be expressed by error model with dynamic. The formulation of fractional model of reference defined in a different order than the plant is far more difficult to analyses and not convergence result is available for a different order fractional adjustment law.

\subsection{Model reference control}

In this problem, it is assumed that the desired output can be modeled by the output of a linear system. We set the following assumptions. 
A1: The unknown system whose input is $u$ and its output is $y$, that $\mathrm{t}$ can be described by a rational or irrational transfer function in the Laplace domain by,

$y / u=P=k_{p} n_{p} / d_{p}$,

where $n_{p}, d_{p}$ are monic polynomials in $\mathfrak{P}$ and $n_{p}$ is Hurwitz (meaning that the zeros of $n_{p}$ are in the left complex plane).

A2: The desired output can be expressed as the output of a system given in the Laplace domain as

$y_{m} / r=M=k_{m} n_{m} / d_{m}$,

where $n_{m}, d_{m}$ are monic polynomials in $\mathfrak{P}, n_{m}, d_{m}$ are Hurwitz polynomials and $r$ is a bounded function of time.

A3: The control input is defined by the linear combination

$u=c_{0} r+c u / \lambda+d y / \lambda$,

where $c_{0}, d, c$ are polynomials to be determined, $\lambda$ is an arbitrary Hurwitz polynomial whose order is to be precised. The linear nature of $u$ is emphasized by writing it as

$u=\theta^{T} w=c_{0} r+\bar{\theta}^{T} \bar{w}$

where $\theta$ is a vector whose components are the coefficients of the polynomials $c_{0}, c$ and $d . w$ is built from $u, y, r$ by using filters of type $s^{\alpha_{i}} / \lambda$.

\subsubsection{Non adaptive problem}

By choosing $\lambda=\lambda_{0} n_{m}$ with $\lambda_{0}$ an arbitrary Hurwitz monic polynomial, the matching equation obtained by imposing that

$y=P u \equiv M r=y_{m}$

implies

$(\lambda-c) d_{p}-k_{p} n_{p} d=c_{0} \frac{k_{p}}{k_{m}} \lambda_{0} n_{p} d_{m}$

which can be satisfied by choosing

$\left\{\begin{array}{l}c_{0}^{*}=k_{m} / k_{p} \\ \lambda_{0} d_{m}=: q d_{p}+t \\ c^{*}=\lambda-q n_{p} \\ k_{p} d^{*}=q d_{p}-\lambda_{0} d_{m}\end{array}\right.$

$A 4:$ if $\operatorname{deg}(\lambda) \geq \operatorname{deg}\left(d_{p}\right)$, then there is no effective derivation in Eq. (13) in the case of noisy output/input measurements. This gives the order of $\lambda_{0}$ and thereby, of $\lambda, c$ and $d$.

\subsubsection{Adaptive problem}

Define $\theta^{*}$ in the same way as vector $\theta$ but now for $d^{*}, c^{*}, c_{0}^{*}$. Let $\phi:=\theta-\theta^{*}$. Since a linear relationship for the input holds, the adaptive goal is translated to the input to get a linear error equation by the following reasoning.

Dividing Eq. (15) by $\lambda d_{p}$ and applying both sides the input $u$, we get (up to initial condition terms associated to stable dynamic $M^{-1}$ and $\left.d^{*} / \lambda\right),\left(c_{0}^{*} M^{-1}+d^{*} / \lambda\right) y=u-c^{*} u / \lambda$. Hence, 
$\bar{\theta}^{*} \bar{w}=u-c_{0}^{*} M^{-1} y$. By replacing Eq. (13) and defining $z^{T}:=\left(M^{-1} y, \bar{w}^{T}\right)$, the following equation is obtained:

$e_{c}:=r-M^{-1} y=\frac{1}{c_{0}} \phi^{T} z$

Note that $e_{c}=M^{-1} y_{m}-M^{-1} y=M^{-1} e_{o}$ or $M e_{c}=e_{o}$. In particular, $e_{c}$ is an available signal. Since $M$ is BIBO stable, if $e_{c}$ converges to zero, then $e_{o}$ also converges to zero. and if $e_{c}$ is bounded, then $e_{o}$ is also bounded.

Some considerations must be taken if $y$ is a noise signal. First, it can be assumed without loss of generality that $M$ has degree zero. Indeed, if the required output is given by $y_{m}=M r$, it can be wrote as $y_{m}=M_{0} M_{s} r=: M_{0} r^{\prime}$ where $M_{0} M_{s}=M, M_{0}$ has degree zero and $r^{\prime}$ is the required reference signal.

A second alternative is filtering. Define $v:=L^{-1} z$ with $L$ a BIBO stable filter such that $M L$ has relative degree zero and $(M L)^{-1}$ is stable (clearly, $L$ always exists by choosing Hurwitz polynomials of suited orders). Similar to the above reasoning, $\bar{\theta}^{-T} L^{-1} \bar{w}=L^{-1}\left(c^{*} u / \lambda+\right.$ $\left.d^{*} y / \lambda\right)$. By dividing Eq. (15) by $\lambda d_{p} L$, we get $\left(c_{0}^{*}(M L)^{-1}+L^{-1} d^{*} / \lambda\right) P=L^{-1}-L^{-1} c^{*} / \lambda$. Then, $\bar{\theta}^{*} L^{-1} \bar{w}=L^{-1} u-c_{0}^{*}(M L)^{-1} y$, which is rewriting as $L^{-1} u=\theta^{* T} v$. Then,

$e_{L}:=\theta^{T} v-L^{-1} u=\phi^{T} v$.

If $\phi \rightarrow 0$ and $v$ is bounded then $e_{L} \rightarrow 0$. By BIBO stability of $L, z$ is bounded, and thus, $e_{o}$ also converges to zero. By the same reasons, if $v, \phi$ are bounded, then $z, e_{o}$ are bounded.

Theorem 3. Assume that hypothesis $A 1-A 4$ are satisfied, $k_{p}<K$ and $K$ is a known constant. If the control parameters are adjusted by

$D^{\alpha} \theta=-\gamma e_{c} z$

where $\gamma \in \mathbb{R}_{\geq 0}, D^{\alpha}$ is the Caputo derivative of order $\alpha, 0<\alpha \leq 1, c_{0}(0)>c_{\min }=k_{m} / K>0$ and whenever $c_{0}=c_{\min }$ and $D^{\alpha} c_{0}<0$ we set $D^{\alpha} c_{0} \geq 0$. Then, the following statements hold

(i) $e_{o}, \theta$ and $u$ remain bounded. Moreover, $I^{\alpha} e_{o}^{2}<\infty$ and the RMS value of $e_{0}$ converges to zero.

(ii) If there exist $k$ such that $z$ has some components in $P E(k, \alpha)$ and the other linearly dependent then $e_{o}$ converges to zero.

Proof. (i) The conditions on $c_{0}$ are an instance of the projection gradient method, whereby $c_{0}>c_{\min } \neq 0$. Then, from (Section 2.3) and Theorem 1 (Remark 2), we have $e_{c} \in \mathcal{L}_{\alpha}^{2}$ and $e_{c}$, $\phi, \theta$ are bounded. From BIBO stability of $M$, we conclude that $e_{o}$ is bounded. Hence, $y$ is also bounded since $y_{m}$ does it.

We prove that $u$ is bounded. $w=\left(r, \bar{w}^{T}\right)^{T}$ with $\bar{w}=\left(\frac{v_{1}^{T}}{\lambda} P^{-1}, \frac{v_{2}^{T}}{\lambda}\right) y=H y$ where $v_{1}, v_{2}$ are vectors of components of type $s^{\alpha_{i}}$. Since $n_{p}$ and $\lambda$ are Hurwitz, $H$ is asymptotically stable. From BIBO stability, $w$ is bounded. Since $\phi$ is bounded, we conclude that $u$ is bounded.

By Theorem 1(i), $e_{c} \in \mathcal{L}_{\alpha}^{2}$. Since $M$ is BIBO stable, its impulse response belongs to $\mathcal{L}^{1}$. By using that $e_{o}=M e_{c}$ and the argument in Section 3.1.3, we have $I^{\alpha} e_{o}^{2}<\infty$. The rest follows from Theorem 1(ii).

(ii) In order to apply Proposition 1 , we must show that $z$ is uniformly continuous. Since $z=\left(M^{-1} P^{-1}, H\right) y, z$ can be realized by a non minimal representation as $D^{q} z_{1}=\Lambda z_{1}+b y$ and $D^{q} z_{2}=\Lambda z_{2}+$ by where $q \leq 1$ is the common commensurate order (by using the proof of Lemma 1 if necessary, see Remark 5.vi). From (i), we conclude that $\left\|D^{q} z\right\|$ is bounded, whereby it is uniformly continuous [31, Proposition 1]. 
Remark 6. (i) Assumption $A 1$ and $A 2$ can be realized by any fractional derivative such that its Laplace transform will be like $s^{\alpha}$, but the adjustment law needs a fractional derivative such that operated with constant functions yields zero. Assumption A3 admits the equivalent representation $u=n_{\pi} / d_{c} r-n_{f} / d_{c} y$ with $n_{\pi}=c_{0} \lambda, d_{c}=\lambda-c$ and $n_{f}=-d$. Assumption A1 is the strongest requiring plant has stable zeros and it is related to the impracticality, even in non-adaptive control, of cancelling unstable zeros (in the pole placement problem this requirement will be not necessary). As shown in the case $\alpha=1$, it is not necessary parameter convergence to achieve the adaptive objective. The non adaptive solution, however, is useful to determine the structure (the order) of the polynomial $c, d$ (assumption A4).

(ii) The error equations are up to a decaying term associated to initial conditions of the (asymptotically stable) transfer functions $M, L$, which appear when $u$ is applied. They were neglected in view of Theorem 1 where the requirement that those terms be $\mathcal{L}_{\alpha}^{2}$ can be assured if they are $\mathcal{L}^{2}$ (see e.g., [32]).

(ii) Experimental verification of the fractional adaptive control of integer system has been performed in [33], using the fractional adjustment Eq. (19). The implementation of integer order adaptive laws always involve software that computes the adaptive parameters and an actuator that applies the control input based on those estimated. Therefore, the difficulties in the implementation of the proposed control will be the same that those of the integer order adaptive laws, but with additional computational load (see Section 6); as shown in [33], the latter are not excessively demanding in computational resources.

\subsubsection{Condition on $\mathrm{r}$ for $w \in P E(n, \alpha)$}

First, we show that if the transfer function $H$ is BIBO stable and of positive relative degree, $H e_{o} \in \mathcal{L}_{\alpha}^{2}$ whenever $e_{0} \in \mathcal{L}_{\alpha}^{2}$ is a bounded function. For $\alpha=1$, it follows since $h \in \mathcal{L}^{1}$ where $h$ is its impulse response and $\left\|f^{*} g\right\|_{2} \leq\|f\|_{2}\|g\|_{1}$. Suppose then that $\alpha<1$. For $H$ of positive relative degree, the initial condition term belongs to $\mathcal{L}^{2}$, by applying the arguments of the proof of [32, Theorem 2]. Hence, it is enough to prove that $h * e_{0} \in \mathcal{L}_{\alpha}^{2}$.

If $f$ is bounded, belongs to $\mathcal{L}_{\alpha}^{1}$ and $g \in \mathcal{L}^{1}$, then $f * g \in \mathcal{L}_{\alpha}^{1}$. Indeed, $I^{\alpha}|g * f|=$ $\int K_{\alpha}(x) \int|f(x-y)||g(y)| d y d x$. By interchanging integrals - which can be done since $g \in$ $\mathcal{L}_{\alpha}^{1}$ by Remark 1 and $f$ is bounded, so the integral is bounded-, we have $I^{\alpha} g * f \leq$ $\int|g(y)| \int K_{\alpha}(x)|f(x-y)| d x d y \leq\left[\int h(y) d y\right] I^{\alpha}\left|e_{0}\right|<\infty$.

Using that $\left(f^{*} g\right)^{r} \leq C f^{* *} g^{q}$ for $r^{-1}=p^{-1}+q^{-1}-1$ with $C:=\|f\|_{p}\left\|_{g}\right\|_{q}$, it follows that $(h *$ $\left.e_{0}\right)^{2} \leq C e_{0}^{2} * h$ with strict inequality if $\left(f^{*} g\right)^{r}$ is bounded and $\|f\|_{p}\left\|_{g}\right\|_{q}$ is unbounded.

An example is if $H$ is commensurate in $\alpha \leq 1$. Indeed, $\eta=H e_{o}$ implies a time realization as $\eta^{(\alpha)}=A \eta+b e_{0}$, the eigenvalues of $A$ with real part lesser than $-\epsilon$ and $b$ a constant vector. Hence $2 V=\eta^{T} \eta$ implies

$$
D^{\alpha} V \leq \eta D^{\alpha} \eta \leq-\epsilon \eta^{T} \eta+|| \eta||\left|e_{o}\right|=-\epsilon\left(\|\eta\|+(2 \epsilon)^{-1}\left|e_{o}\right|\right)^{2}+\left(2 \epsilon^{2}\right)^{-1}\left|e_{o}\right|^{2} \leq C e_{o}^{2},
$$

where $C=\left(2 \epsilon^{2}\right)^{-1}$. By integrating, $V(t)-V(0) \leq C I^{\alpha} e_{o}^{2}$, we conclude that $V$ is bounded. Moreover, $\quad \infty>-V(t)+V(0)+C I^{\alpha}\left|e_{o}\right|^{2} \geq I^{\alpha} \epsilon\left(\|\eta\|+(2 \epsilon)^{-1}\left|e_{o}\right|\right)^{2} \geq \epsilon I^{\alpha}(\|\eta\|)^{2}$. Thus, $\eta \in \mathcal{L}_{\alpha}^{2}$.

Let $w_{m}:=H y_{m}$. Then $w-w_{m}=H e_{o}$. If $e_{0}^{2} \in \mathcal{L}_{\alpha}^{1}$ and $h \in \mathcal{L}^{1}$, then $h * e_{0} \in \mathcal{L}_{\alpha}^{2}$. From Theorem 3(i) we have that $e_{o} \in \mathcal{L}_{\alpha}^{2}$. If $H$ is BIBO stable and has positive relative degree, then from the proof of Theorem 3(i), we have that $w-w_{m}=H e_{0} \in \mathcal{L}_{\alpha}^{2}$. By using Theorem 1, we know that the convergence condition is not affected if we only consider $w_{m}$. 
Since $w_{m}=H y_{m}=H M r$, if $r$ is sufficiently rich order $n=\operatorname{dim}(w)$, namely if contains at least $n$ spectral frequencies, then $w_{m} \in P E(n, \alpha)$, whenever $H, M$ are commensurate (see [16, Property 11]). In particular, if $w_{m} \in P E(n, \alpha)$ then $w \in P E(n, \alpha)$.

Consider again the relation $w_{m}=H M r$ with $H M$ BIBO stable. If $r$ is a constant or a step function, $w_{m}$ converges to a constant. Then $w_{m}$ has a component $P E(1)$ and the rest $(n-1)$ 1.d. up to decaying terms (see Example 1). Hence, according to Proposition $1, \lim _{t \rightarrow \infty} e_{o}(t)=0$ whenever $r$ is a step function.

\subsection{Pole placement control}

In this problem, it is only relevant to change the poles of the unknown system. The method of model reference control can be used by taking again $M=n_{m} / d_{m}$ but it changes the zeros of the plant. Moreover, the requirement $n_{p}$ to be Hurwitz in $A 2$ will be not necessary. Consider the following assumptions.

B1: The unknown system can be realized by a transfer function in the Laplace Domain

$y / u=P=n_{p} / d_{p}$,

where $n_{p}, d_{p} \in \mathfrak{P}, d_{p}$ is monic and $n_{p}, d_{p}$ are coprimes.

B2: The required poles are the zeros of $d_{m} \in \mathfrak{P}$ a Hurwitz monic polynomial.

B3: The control signal has the following structure

$u=r+c u / \lambda+d y / \lambda$

where $c, d \in \mathfrak{P}$ are to be determined, and $\lambda \in \mathfrak{P}$ is arbitrary Hurwitz.

\subsubsection{Non adaptive problem}

By defining $z:=u / d_{p}=y / n_{p}$, we get from Eqs. (20) and (21)

$\left[\lambda d_{p}-d n_{p}-c d_{p}\right] z=\lambda r$.

The equation

$d n_{p}+c d_{p}=\lambda\left(d_{p}-d_{m}\right)$,

on $c, d$ has solutions $c^{*}, d^{*}$ by equating order at both sides. Alternatively, by coprimality between $n_{p}, d_{p}$ and using Lemma 1 , the equation $d_{0} n_{p}+c_{0} d_{p}=1$ has solution and, by taking $d=\lambda\left(d_{p}-d_{m}\right) d_{0}$ and $c=\lambda\left(d_{p}-d_{m}\right) c_{0}$, we have a solution for Eq. (23).

By replacing Eq. (23) in Eq. (22), we obtain $z=r / d_{m}$, i.e., $y=r n_{p} / d_{m}$, i.e., the poles of the plant were changed.

\subsubsection{Adaptive problem}

Define $\bar{z}:=z / \bar{\lambda}, \bar{u}:=u / \bar{\lambda}$ and $\bar{y}:=y / \bar{\lambda}$ with $\bar{\lambda}$ an arbitrary Hurwitz polynomial. Multiplying Eq. (23) by $\bar{z}$, we obtain the equation

$d \bar{y}-c \bar{u}+\lambda d_{m} \bar{z}=\lambda \bar{u}$,

which is linear in the unknown parameters $(c, d)$. Choose polynomials $e, f$ such that

$e n_{p}+f d_{p}=1$,

which, by coprimality between $n_{p}, d_{p}$ and Lemma 1 , there always exist (say $e^{*}, f^{*}$ ). Note that $\bar{z}=e \bar{y}+f \bar{u}$ whereby Eq. (24) can be written as

$d \bar{y}-c \bar{u}+e \lambda d_{m} \bar{y}+f \lambda d_{m} \bar{u}=\lambda \bar{u}$ 
Defining $\theta^{*}$ as the vector containing the coefficients of polynomials $c^{*}, d^{*}, e^{*}, f^{*}$ and $w$ defined from $u, y$ through filters of type $s^{\alpha_{i}} / \bar{\lambda}$.

B4: Consider $\operatorname{deg}(\bar{\lambda}) \geq \max \left\{\operatorname{deg}(d), \operatorname{deg}(c), \operatorname{deg}\left(e \lambda d_{m}\right), \operatorname{deg}\left(f \lambda d_{m}\right)\right\}$.

Thus, we can write the above equation as

$\theta^{* T} w=\lambda \bar{u}$

Consider the minimization of $e_{c}^{2}:=\left(\theta^{T} w-\lambda \bar{u}\right)^{2}$. By noting that

$e_{c}=\theta^{T} w-\lambda \bar{u}=\theta^{T} w-\theta^{* T} w=\phi^{T} w$,

we can use the continuous adjustment $D^{\alpha} \phi=-\left(\theta^{T} w-\lambda \bar{u}\right) w=-e_{c} w$

Theorem 4. Let us assume that hypothesis B1-B4 hold. Consider the adjustment

$D^{\alpha} \theta=-e_{c} \frac{w}{1+w^{T} w}$

where $0<\alpha \leq 1$ and $D^{\alpha}$ is Caputo Derivative. If $\frac{w}{1+w^{T} w} \in P E(l)$ with $l=\operatorname{dim}(\theta)$ then $\lim _{t \rightarrow \infty} \theta=\theta^{*}$, all variables remain bounded, and the desired poles given by $d_{m}$ are asymptotically reached.

Proof. From Theorem 1, we have that $\theta \rightarrow \theta^{*}$, that is, $c \rightarrow c^{*}$ and $d \rightarrow d^{*}$. On the other hand, if $c=c^{*}$ and $d=d^{*}$ then the closed loop is a time-invariant linear system with the required poles. Moreover, since at each instant the closed loop can be seen as a linear system, the closed loop admits a representation by pseudo state as in Eq. (9) for some commensurate order (see Lemma 1), where $A=A\left(\theta^{*}\right), \delta A(t)=\delta A\left(\theta(t), \theta^{*}\right), \delta A(t) \rightarrow 0$ as $t \rightarrow \infty$ and $v \equiv 0$. From Theorem 2 , we conclude boundedness of all variables and the desire behavior is asymptotically reached.

\section{Indirect adaptive control}

In this approach an asymptotic estimation of the unknown linear system's parameters is done. The controller is built from the non adaptive solution of each control problem (model reference, pole placement, compensator or other adaptive problem) by replacing with those estimates. For a linear time-invariant system with general order derivative, this estimation can be posed as Eq. (4), allowing us to introduce fractional adjustments even for integer order systems.

Consider the unknown system realized in Laplace domain by

$y=\frac{\sum_{i=0}^{m} b_{i} s^{\beta_{i}}}{\sum_{i=0}^{n} a_{i} s^{\alpha_{i}}} u$

where $\alpha_{0}<\cdots<\alpha_{n}, \beta_{0}<\cdots<\beta_{n}$ with $a_{n} \neq 0$ and without loss of generality, it is assumed $a_{n}=1$. To avoid the effect of noise perturbations, it is defined, for a Hurwitz polynomial $\lambda$ of degree at least $\max \left\{\alpha_{n}, \beta_{n}\right\}, \bar{y}=y / \lambda, \bar{u}=u / \lambda$. Then,

$\bar{y}=\frac{\sum_{i=0}^{m} b_{i} s^{\beta_{i}}}{\sum_{i=0}^{n} a_{i} s^{\alpha_{i}}} \bar{u}$

This system can be realized in time domain by any generic fractional derivative (i.e., a derivative such that its Laplace transform holds for any function $f$ that $\mathcal{L}\left[D^{\alpha} f\right](s)=s^{\alpha} f+$ 
$F(0, s)$ where $F$ is initial condition term) by the following equation

$\sum_{i=0}^{n} a_{i} D^{\alpha_{i}} \bar{y}=\sum_{i=0}^{m} b_{i} D^{\beta_{i}} \bar{u}$

By defining $w:=\left(\bar{y}, D^{\alpha_{1}} \bar{y}, \ldots, D^{\alpha_{n-1}} \bar{y}, \bar{u}, D^{\beta_{1}} \bar{u}, \ldots, D^{\beta_{m}} \bar{u}\right)^{T}:[0, \infty) \rightarrow \mathbb{R}^{n+m+1}$ and $\theta:=$ $\left(-a_{0},-a_{1}, \ldots,-a_{n-1}, b_{0}, \ldots, b_{m}\right)^{T} \in \mathbb{R}^{n+m+1}$, we get

$D^{\alpha_{n}} \bar{y}=\theta^{T} w$

Let $e_{i}:=\hat{\theta}^{T} w-D^{\alpha_{n}} \bar{y}$ to be a measure of the identification error.

Theorem 5. Consider system (31) and $u$ rich of $\operatorname{order} l=\operatorname{dim}(\theta)$.

(i) If the parameters are adjusted by

$\left\{\begin{array}{l}\dot{\hat{\theta}}=-\Gamma e_{i} w \\ \dot{\Gamma}=. \Gamma w w^{T} \Gamma \\ \Gamma\left(t_{i}\right)=I\end{array}\right.$

where $t_{i+1}:=t_{i}+\delta_{i}$ with $\delta_{i}:=\operatorname{argmin}_{\Delta} \int_{t_{i}}^{t_{i}+\Delta} w w^{T} d \tau \geq \epsilon I$, then $\lim _{t \rightarrow \infty} \hat{\theta}(t)=\theta$.

(ii) Consider the following adjustment

$D^{\alpha} \hat{\theta}=-e_{i} \frac{w}{1+w^{T} w}$,

where $0<\alpha \leq 1$. Then, $\lim _{t \rightarrow \infty} \hat{\theta}(t)=\theta$.

(iii) By replacing $\hat{\theta}$, estimated with (i) or (ii), in the non adaptive solution of Model Reference or Pole Placement, the objective of the control is reached asymptotically. Moreover, $e_{i}$ converges to zero.

Proof. (i) Since $u$ is rich of order $l$ then $w \in P E(l)$ [16]. By definition, $D \hat{\theta}=-\Gamma\left(\theta^{T} w-\right.$ $\left.\hat{\theta}^{T} w\right) w=-\Gamma w w^{T} \phi$ where $\phi:=\theta-\hat{\theta}$. By applying [3, Lemma A3], we conclude that $\phi$ converges to zero.

(ii) Similar to (i) but using Proposition 1.

(iii) By replacing $\hat{\theta}$ in the non adaptive solution of the controllers of Section 3 , we have that $\phi \rightarrow 0$ since $\hat{\theta} \rightarrow \theta$. The linear relationships deduced in Section 3 are such that the convergence of $e_{c}$ to zero implies the convergence of $e_{o}$ to zero or that the desired poles are asymptotically achieved. Therefore, it remains to prove that $w$ is bounded. In all cases, we can define $w_{m}:=H r$ where $H=H\left(\theta^{*}\right)$ is the asymptotically stable vector transfer function associated to the transfer function $M$ or to the required poles, where the dependence of $\theta^{*}$ is continuous. Since $H(\hat{\theta})$ converges to $H\left(\theta^{*}\right)$, by the respective matching equation, we can express (commensurate in the same order $q$, if necessary, the transfer function $H\left(\theta^{*}\right)$ ) the following equation relating $r(t)$ to $w(t)$ as

$\left\{\begin{array}{l}D^{q} z(t)=(A+\delta A(t)) z(t)+(b+\delta b(t)) r(t) \\ w(t)=(c+\delta c(t)) z(t)\end{array}\right.$

where $\|\delta A(t), \delta b(t), \delta c(t)\|$ is bounded and converging to zero. This equation relating $r(t)$ to $w(t)$ follows the idea of [3, II.B]. By Theorem 2, we conclude that $w$ is bounded provided that $r$ is bounded.

Remark 7. (i) For other adaptive problems, the indirect method always can be expressed by an Error Model of Type I in the estimation part. In particular, the compensator strategy can be utilized following the developments in [16]. 
(ii) Note that if $c=c^{*}, d=d^{*}$ in the non adaptive solution, there exists a stable timeinvariant scalar filter $H^{*}$ such that $u_{m}:=H^{*} r$. If $r$ contains $l$ spectral frequencies, then $u_{m}$ also contains $l$ spectral frequencies ([16, Property 11]). In particular if $r$ is rich of order $l$ then $u_{m}$ is rich of order $l$. But $u=\theta^{T} w$ differs by a term $\mathcal{L}_{\alpha}^{2}$ from $u_{M}=\theta^{* T} w$, since for any continuous differentiable $u, e_{i} \in \mathcal{L}_{\alpha}^{2}$. From Theorem 1, we can conclude that if $r$ is rich of order $l$ then $u$ assures convergence.

\section{Robust behavior}

We establish a simple robustness advantage of fractional gradient adjustment in comparison with the usual integer one. Advantages by using a fractional order reference model for integer order plants were suggested by simulations in [9] regarding the transient behavior and the stability. The latter is roughly related to the enlargement of stability region for fractional linear time-invariant systems which allows to choose reference model in a larger set, meanwhile the former is roughly due to the integral definition of fractional derivative. We generalize the result [35] where robustness was established for the restricted set of functions $P E(n)$.

In considering perturbations which take into account noise, unmodeled dynamics, initial conditions terms, we are relaxing the linear time-invariant assumption of Sections 3 and 4.

We note from Sections 3 and 4 that global boundedness of the controlled system follows from boundedness of $e, \phi$. Hence, the robustness behavior of the controlled adaptive system follows directly from robustness of the adaptive scheme.

\subsection{Intrinsic robustness}

By Theorem 1, we have that the $\alpha$-adjustment given by Eq. (4) is robust under additive perturbations $v \in \mathcal{L}_{\alpha}^{2}$ in the error measurement, namely

$e=\phi^{T} w+v$

By Remark 1 and [27, Proposition 4], it follows that $\mathcal{L}_{1}^{2} \subset \mathcal{L}_{\alpha}^{2}$ for $0<\alpha<1$. Hence, fractional adjustment in comparison with integer adjustment presents this enlargement of non destabilizing signals. Not that any perturbation vanishing after a time $T$ belongs to $\mathcal{L}^{2}$.

More generally, consider $v$ a bounded differentiable function and than $w$ is bounded or that has been normalized (e.g., $w / \sqrt{1+w^{T} w}$ ). We will show that $e$ is always bounded, though $\phi$ could diverge.

From [29, Lemma 1], Eq. (34) and the same parametric adjustment of Eq. (4), we get $2^{-1} D^{\alpha}\left[\phi^{T} \phi\right] \leq \phi^{T} D^{\alpha} \phi \leq-\epsilon^{2}+\epsilon \nu$,

where $\epsilon=\phi^{T} w$. Suppose than $\epsilon$ is unbounded, then there exists $T$ such that for all $t>T$, $\epsilon^{2}(t)>C$ where $C$ is a constant number that makes $-\epsilon^{2}+\epsilon \nu<0$ for all $\epsilon^{2}>C$. Therefore, we have that for all $t>T, D^{\alpha} V \leq 0$. By [31, Theorem 8(i)] $\phi$ is bounded, whereby $\epsilon$ is bounded (since $w$ is bounded), which is a contradiction. Then $\epsilon$ and hence $e$ are bounded, whenever $v$ is bounded.

Remark 8. (i) For Error Model of Type II with same order, namely

$$
\left\{\begin{array}{l}
D^{\alpha} e=A e+\phi^{T} w+v \\
D^{\alpha} \phi=-e w
\end{array}\right.
$$


Then, $2 D^{\alpha}\left[\phi^{T} \phi+e^{T} e\right] \leq-e^{T} Q e+e^{T} P v$, for suited $P>0, Q>0$ provided that $A$ is stable in the integer sense and the same procedure as above shows that $e$ is always bounded.

(ii) That $\phi$ can diverge even though $e$ remains bounded is shown for instance for the integer Error Model II in [30] and for the fractional Error model I in [35].

(iii) The above reasoning allows to generalize [31, Theorem 8(ii)]. Consider a system of trajectories $x(\cdot)$ for which a non negative function $V=V(x)$ is defined such that $D^{\alpha} V(x(t)) \leq f(x, t)$. Consider $x=\left(x_{s}^{T}, x_{r}^{T}\right)^{T}$ where $x_{s}:[0, \infty) \rightarrow \mathbb{R}^{s}$. If the set $\Omega=\left\{x_{s} \in \mathbb{R}^{s}\right.$ : $\left.D^{\alpha} V(x) \geq 0\right\}$ is compact in $\mathbb{R}^{s}$ then $x$ has its first $s$ coordinates bounded.

(iv) In [35], it was proved that for a bounded perturbation, boundedness of $e, \phi$ can be assured if $w \in \operatorname{EP}(n)$ where $n=\operatorname{dim}(\phi)$.

Boundedness of $\phi$ can be assured for more general perturbations by modifying the adaptive law. We examine some of those modifications, which are generalization to fractional order from those of $[1,2]$.

\subsection{Projection modification}

By using the gradient method with projection (\$2.3) and Remark 4, we obtain boundedness of $\theta$ (and therefore, boundedness of $e$ ) if a priori bound (say) $c$ of $\left\|\theta^{*}\right\|$ is known; the set $C$ being defined as $\left\{x \in \mathbb{R}^{n}:\|x\|<c\right\}$. The notable advantage, is that we can assure that if the perturbation never occurs or it vanishes (module $\mathcal{L}_{\alpha}^{2}$ ), the conditions of convergence of the error are the same that in Theorem 1.

\section{3. $\sigma \theta$ modification}

Consider the following modification to Eq. (4)

$D^{\alpha} \phi=-e w-\sigma \theta$

where $\sigma>0$ is a constant number. This modification is called $\sigma \theta$ when $\alpha=1$ ([2, Chapter 8]).

Then, using that $\phi=\theta-\theta^{*}$, we have

$\left(D^{\alpha} \phi^{T} \phi\right) / 2 \leq \phi^{T} D^{\alpha} \phi \leq-\left(\phi^{T} w\right)^{2}-v \phi^{T} w-\sigma\|\phi\|^{2}-\sigma \phi^{T} \theta^{*}$

Since $w, \theta^{*}$ are bounded, the set $\left\{\phi \in \mathbb{R}^{n}:\left(\phi^{T} w\right)^{2}+\nu \phi^{T} w+\sigma\|\phi\|^{2}+\sigma \phi^{T} \theta^{*} \leq 0\right\}$ is bounded (if it were unbounded the positive terms $\left(\phi^{T} w\right)^{2}, \sigma\|\phi\|^{2}$ would dominate and the inequality does not hold) and closed (since the inequality depends continuously on $\phi$ ), hence it is compact. By applying [31, Theorem 8] we conclude that $\phi$ is bounded, whereby $e$ is also bounded.

This modification is also robust for parametric variations. Indeed, consider $\theta^{*}(t)$ differentiable function, $\left\|\theta^{*}(t)\right\|<C_{1},\left\|D^{\alpha} \theta^{*}(t)\right\|<C_{2}$ and $v=0$ (the same claim holds for $v$ bounded). We have

$D^{\alpha} \phi=-e w-\sigma \theta+D^{\alpha} \theta^{*}$

and hence,

$\left(D^{\alpha} \phi^{T} \phi\right) / 2 \leq-\left(\phi^{T} w\right)^{2}-\sigma \phi^{T} \phi-\sigma \phi^{T} \theta^{*}+\phi^{T} D^{\alpha} \theta^{*}$.

The set $\left\{\phi \in \mathbb{R}^{n}:\left(\phi^{T} w\right)^{2}+\sigma \phi^{T} \phi+\sigma \phi^{T} \theta^{*}-\phi^{T} D^{\alpha} \theta^{*}(t) \leq 0\right\}$ is bounded and closed, hence it is compact. By applying [31, Theorem 8] we conclude that $\phi$ is bounded, whereby $e$ is also bounded. 
But the condition $D^{\alpha} f<C$ is stronger than $D f$. In fact, note first that if $\|D f\|<C$ it does not implies $\left\|D^{\alpha} f\right\|<C$ (just take a function of constant growth whereby $D^{\alpha} f$ diverges). However since $\theta^{*}(t)$ is a differentiable function $\left\|D^{\alpha} f\right\|<C$ implies $\|D f\|<C$ (by contradiction).

Therefore, when $\alpha=1$, Lipschitzian variations are not destabilizing but when $\alpha<1$ the set was proved more restricted than Lipschitzian.

\section{Simulations}

The aim of this section is to illustrate in the proposed methods of the mixed order approach three ways to control integer order systems using fractional calculus, to validate the theorems and to find advantages and to discuss them. We study them separately to distinguish the specific contribution in each case, though combinations of them are possible. The discussion is oriented to find optimal differentiation order in the controller design. Fractional operator is simulated in MATLAB/Simulink software using approximations of $s^{\alpha}$ through integer order linear filters; hence, there is an increase of the computational load in comparison with non mixed strategy.

Consider the unstable plant

$y_{p}=\frac{b}{s+a} u$,

where $a=-1$ and $b=1$-which are supposed to be unknown- and the BIBO stable reference model

$y_{m}=\frac{1}{s+1} r$

with $r$ a function yielding a desired trajectory $y_{m}$. According to Eq. (16) with $\lambda_{0}=1$, the control parameters are $c_{0}=1, q=1, \theta_{1}:=c=1-b, \theta_{2}:=d=a-1, \lambda=1$ and replacing in Eq. (13), the control is given by

$u=r+\theta_{1} u+\theta_{2} y_{p}$.

By Eq. (19), the parameters can be adjusted using

$\left\{\begin{array}{l}D^{\alpha} \theta_{1}=-e_{c} u \\ D^{\alpha} \theta_{2}=-e_{c} y_{p}\end{array}\right.$

where $0<\alpha \leq 1$.

Fig. 1(a),(b) and 1(c),(d) show the tracking feature of the adaptive control when $r$ is a step function and a sum of sinusoidal of 4 spectral frequencies, respectively. Fig. 1(a) shows -according to Theorem 3(i) and Section 3.1.3- the convergence of the error. It is seen that the lesser the $\alpha$ the slower is the response, yielding a smoother transient for the smallest $\alpha$. Fig. 1(b) shows that the controller parameters do not converge to the ideal ones since $r$ is not rich enough, but Fig. 1(d) shows this convergence (to $\theta_{1}^{*}=0$ and $\theta_{2}^{*}=-2$ ), according to Theorem 3(ii) and Section 3.1.3, when $r$ is rich enough (in this case a sinusoidal of arbitrary frequency). In Fig. 1(c), the best performance in transient and speed is obtained for $\alpha=0.9$. Fig. 1(e) and 1(f) present the control signals for the step and a sinusoidal references respectively; in the first one the minimal (in absolute value) control is achieved for $\alpha=0.7$, and in the second for $\alpha=1$.

Consider now the reference model

$y_{m}=\frac{1}{s^{\beta}+1} r$. 


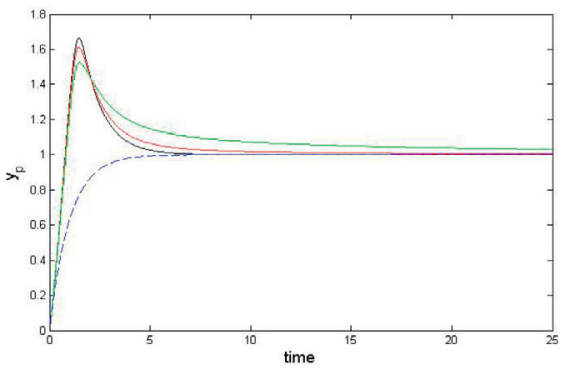

(a) Step reference

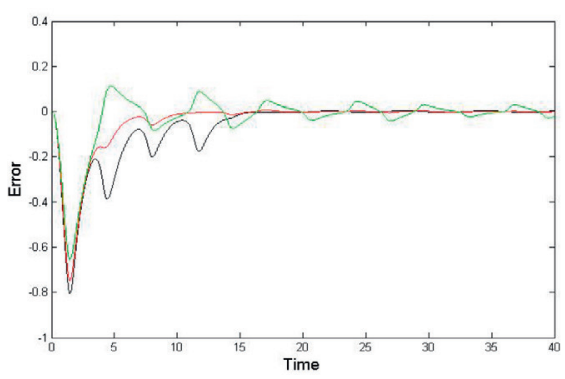

(c) Sinusoidal reference

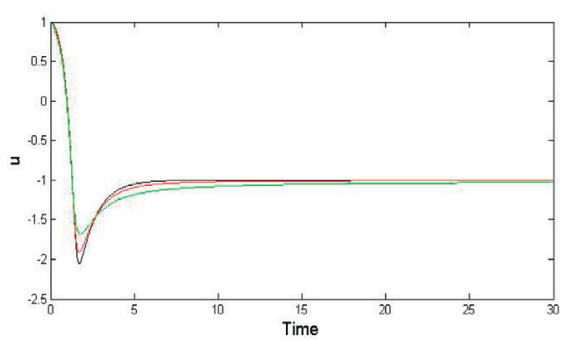

(e) Step reference

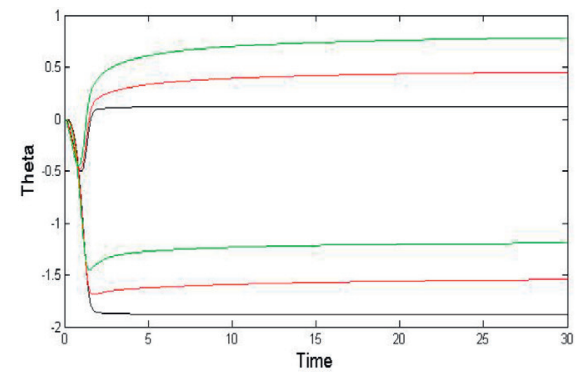

(b) Step reference

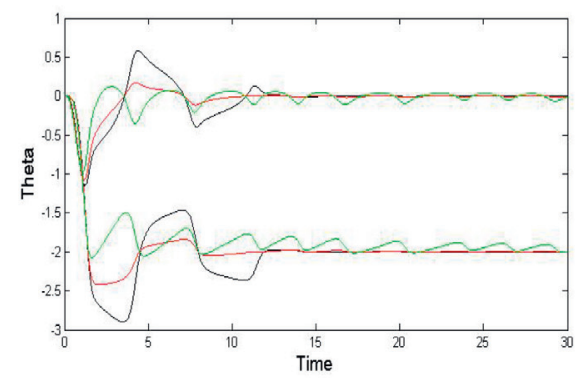

(d) Sinusoidal reference

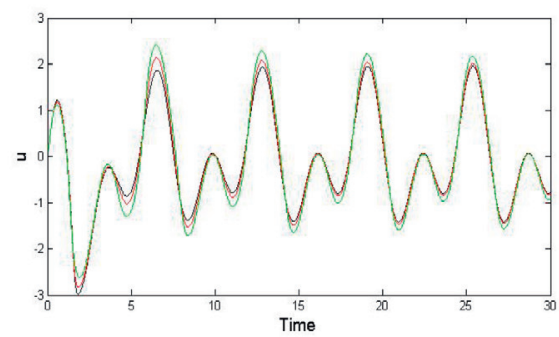

(f) Sinusoidal reference

Fig. 1. Controlled system evolution. Green line $\alpha=0.7$, red line $\alpha=0.9$, black line $\alpha=1$, dashed line $y_{m}$. In $(b)$, (d) the upper line is $\theta_{1}$. (For interpretation of the references to color in this figure legend, the reader is referred to the web version of this article.).

According to Eq. (16), the control parameters with $\lambda_{0}=s+1$ are given by $c_{0}=1 ; q=$ $s^{\beta}, t=s+(1-a) s^{\beta}+1 ; c=s-b s^{\beta}+1 ; d=-t ; \lambda=s+1$. Therefore, the adaptive control (13) has the following structure,

$u=r+\left(s+\theta_{1} s^{\beta}+1\right) \frac{u}{s+1}+\left(-s+\theta_{2} s^{\beta}-1\right) \frac{y}{s+1}$.

The parameters $\theta_{1}, \theta_{2}$ are adjusted with Eq. (19). We perform the following study. Suppose that we want to follow a step signal equals to 1 . We can choose any fractional or integer stable filter (with $k_{m}=1$ ) as a model since by taking $r$ a step function, $y_{m}$ will converge to a constant value ( 1 in this case) and our adaptive control objective is $y_{p} \rightarrow y_{m}$ (achieved by Theorem 3 and Section 3.1.3). In terms of model (40), $\beta$ can take any value in $(0,1]$. In 


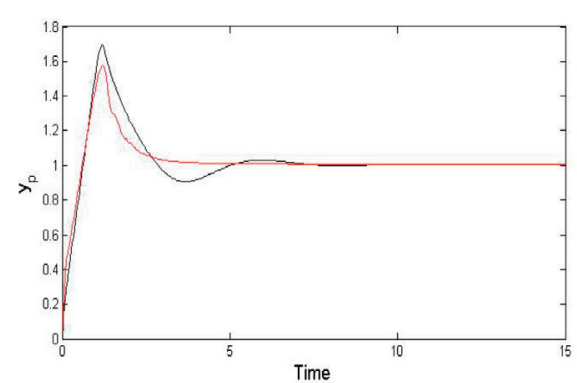

(a) Plant output

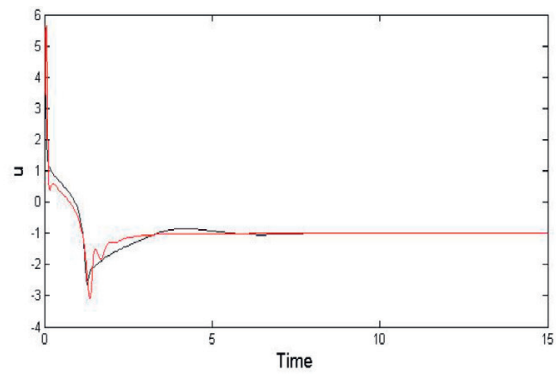

(b) Control input

Fig. 2. Evolution of the controlled plant and the control function. Red line $\beta=0.8$, black line $\beta=1$. (For interpretation of the references to color in this figure legend, the reader is referred to the web version of this article.).

Fig. 2(a),(b), we compare the output and control behavior for $\beta=1$ and $\beta=0.8$ using $\alpha=1$ in the adjustment law (19) in both cases. It is noted a better output performance of the fractional model control in transient and speed in Fig. 2(a) and a similar behavior in the control signals as shown in Fig. 2(b). Though at the initial times the fractional model control have larger variation than the integer one, the eventual associated practical problem of input saturation is not present according to the arguments given in [1, p. 121].

In the last study, we use the pole placement design (Section 3.2) together with the indirect adaptive estimation (Section 4) to illustrate Theorem 5. The reason for this choice is that the controller parameters of Theorem 3 would be $>2$ but in the indirect approach it is only required 2. Again we consider system (36) with $a=-1$ but we also consider $a=1$ since the problem is pole placement. The desired poles are given by $s^{\beta}+1$ for $\beta \in(0,1]$. According to Eq. (21), (23) and taking $\lambda=1$, the matching control is given by

$u=\frac{1}{2} r+\frac{1}{b}\left(2 s-2 s^{\beta}-2+a\right)$.

According to Section 4, the indirect adaptive control is given by Eq. (42) with the estimations $\hat{a}, \hat{b}\left(-\theta_{1}, \theta_{2}\right.$ according to Eq. (30)) instead of $a, b$, respectively. Note that in the implementation, the actualization of Eq. (42) must occur only when $\hat{b} \neq 0$ and naturally $\hat{b}(0) \neq 0 \quad(\hat{b}(0)=0.1$, in our simulation). To test the effectiveness, we use as reference a step function because as the zeros are not modified in the method of Section 3.2, a perfect matching must occur since the high frequency gain of $n_{p} /\left(s^{\beta}+1\right)$ is 1 . Through Eqs. (31) and (33), the parameters are estimated with $\alpha=1$. However, by Theorem 5 and Remark 4, the reference must have at least 4 spectral frequencies. This difficulty is solved by a period of estimation previous to the application of the step function reference $(T=30$ in the simulation). According to [16], the speed of parameter convergence roughly is larger if the amplitude of the reference is larger; whereby, a trade off between $T$ and such an amplitude is involved. Fig. 3 shows the indirect adaptive controlled output and the parameter convergence for $a= \pm 1$ and $\beta=0.9$. In Table 1 , the RMS value of $y_{p}$, when white noise is added in the feedback loop, is computed for the cases of $\beta=1$ and $\beta=0.8$. The best performance of the fractional poles gives a slight justification to this assignment for an integer order plant. 


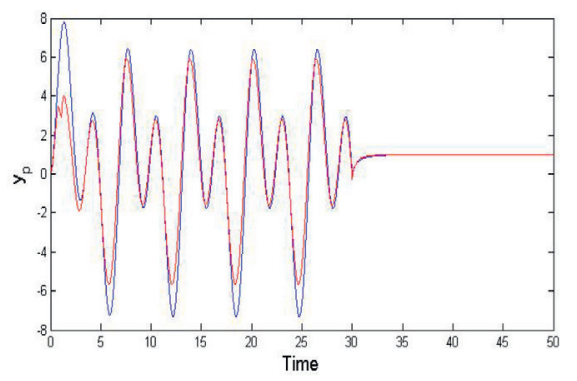

(a) Blue line is the reference, red line is $y_{p}$ when $a=1$

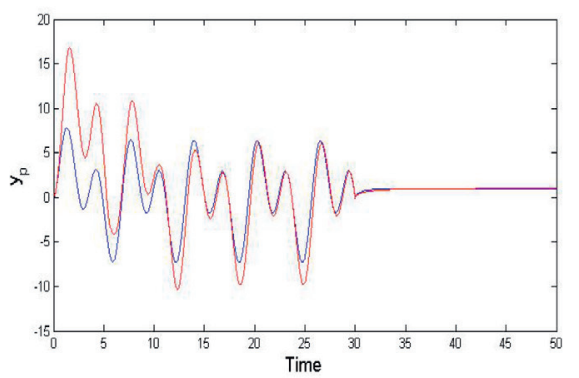

(c) Blue line is the reference signal, red line is $y_{p}$ for $a=-1$

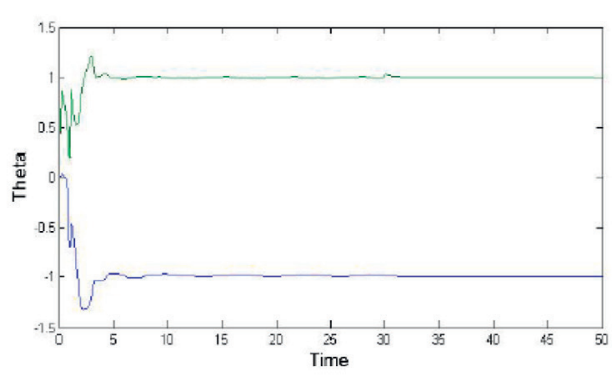

(b) $\theta(t)$ for $a=1$. The blue line is $\theta_{1}$

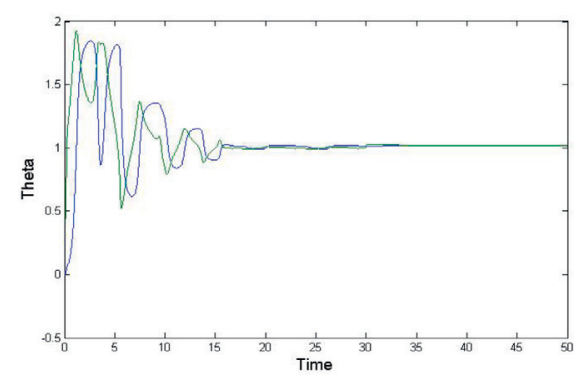

(d) $\theta(t)$ for $a=-1$. The blue line is $\theta_{1}$

Fig. 3. Evolution of the plant and the parametric estimation using indirect pole placement control.

Table 1

Pole placement: RMS value of $y_{p}$.

\begin{tabular}{lll}
\hline Noise power & $\beta=1$ & $\beta=0.8$ \\
\hline 0.1 & 1.186 & 1.177 \\
0.01 & 1.058 & 1.051 \\
0.001 & 1.015 & 1.005 \\
\hline
\end{tabular}

\section{Conclusions}

We have established main lemmas and theorems for adaptive control designs in the more general perspective of mixed order control. We exemplified how to apply them in MRAC and pole placement adaptive problems; in particular, there is at least three ways to introduce fractional calculus in controlling an integer order system: firstly, by choosing a fractional model of reference; secondly, by choosing a fractional adjustment law and the third is choosing a fractional filter $\lambda$ for the inputs. We discuss theoretical and simulation advantages of mixed order strategy to justify its application in adaptive control of integer system.

It was shown that the Error Model of Type I simplifies the analysis and formulation required in mixed order adaptive control. For other adaptive problems, the application of Theorems 1 remains valid provided that the problem can be formulated in this error model. 


\section{Conflict of interest}

The authors declare that there is no conflict of interest regarding the publication of this paper.

\section{In memoriam}

This paper is dedicated to the memory of Doctor Nicolas Humberto Beltrán Maturana, Professor at the Electrical Engineering Department of the University of Chile. Nicolas was an outstanding researcher and a better person, who unexpectedly passed away on December 2, 2014. It has been a sensible loss for his family, friends, students, and colleagues. R.I.P.

\section{Acknowledgments}

This work has been supported by CONICYT- Chile, under the grants FB0809 "Centro de Tecnología para la Minería” and FONDECYT 1150488 "Fractional Error Models in Adaptive Control and Applications".

\section{References}

[1] S. Sastry, M. Bodson, Adaptive control: Stability, Convergence and Robustness, Prentice Hall, 1994.

[2] K.S. Narendra, A.M. Annaswamy, Stable Adaptive Systems, Dover Publications, 2005.

[3] E. Bai, S. Sastry, Global stability proofs for continuous-time indirect adaptive control schemes, IEEE Trans. Autom. Control 32 (6) (1987) 537-543.

[4] H. Elliott, R. Cristi, M. Das, Global stability of adaptive pole placement algorithms, IEEE Trans. Autom. Control 30 (4) (1985) 348-356.

[5] N. Wang, T. Zhang, Y. Yi, Q. Wang, Adaptive control of output feedback nonlinear systems with unmodeled dynamics and output constraint, J. Frankl. Inst. 354 (13) (2017) 5176-5200.

[6] A. L'Afflitto, Differential games, Partial-state stabilization, and model reference adaptive control 354 (1) (2017) 456-478.

[7] T. Wang, J. Qiu, H. Gao, Adaptive neural control of stochastic nonlinear time-delay systems with multiple constraints, IEEE Trans. Syst. Man Cybern. (2016), doi:10.1109/TSMC.2016.2562511.

[8] W. Deng, J. Yao, D. Ma, Robust adaptive asymptotic tracking control of a class of nonlinear systems with unknown input dead-zone, J. Frankl. Inst. 352 (12) (2015) 5686-5707.

[9] B.M. Vinagre, I. Petra, I. Podlubny, Y.Q. Chen, Using fractional order adjustment rules and fractional order reference models in model-reference adaptive control, Nonlinear Dyn. 29 (1) (2002) 269-279.

[10] S. Ladaci, Y. Bensafia, Indirect fractional order pole assignment based adaptive control, Eng. Sci. Technol. 19 (1) (2016) 518-530.

[11] S. Ladaci, S. Chettah, K. Khettab, Direct fractional adaptive pole placement control for minimal phase systems, in: Proceedings of the Fifteenth International Conference on Sciences and Techniques of Automatic Control and Computer Engineering, 2015.

[12] J. Chen, Distributed containment control of fractional-order uncertain multi-agent systems, J. Frankl. Inst. 353 (7) (2016) 1672-1688.

[13] C. Huan, Y. Li, D. Liu, X. Guan, Stability analysis for fractional-order PD controlled delayed systems, J. Frank1. Inst. 353 (13) (2016) 3118-3132.

[14] I. Podlubny, Fractional order systems and PIlambda $d^{m u}$ controller, IEEE Trans. Autom. Control 442 (1999) 208-214.

[15] F. Merrikh-Bayat, Fractional-order unstable pole-zero cancellation in linear feedback systems, J. Process Control 23 (6) (2013) 817-825.

[16] J.A. Gallegos, M. Duarte-Mermoud, Convergence of fractional adaptive systems using gradient approach, ISA Trans. 69 (2017) 31-42.

[17] T. Mora, Solving Polynomial Equation Systems I, Encyclopedia of Mathematics and its Applications, 99, Cambridge University Press, 2003. 
[18] H. Cong, Q. Zhidong, M. Qian, Z. Xi, Factional order model reference adaptive control based on Lyapunov stability theory, in: Proceedings of the Thirty-Fifth Chinese Control Conference, Chengdu, China, 2016, pp. $27-29$.

[19] S. Bao, Y. Jian, D. Chao, On fractional model reference adaptive control, Sci. World J. 2014 (2014) Article ID 521625,8 pages.

[20] M.L. Corradini, R. Giambo, S. Pettinari, FO sliding surface for the robust control of integer-order LTI plants, IFAC Proc. Vol. 47 (3) (2014) 2034-2039.

[21] J.G. Lu, Y.Q. Chen, Robust stability and stabilization of fractional-order interval systems with the fractional-order $\alpha: 0<\alpha<1$ case, IEEE Trans. Autom. Control 55 (1) (2010) 152-158.

[22] Z. Gao, Robust stabilization criterion of fractional-order controllers for interval fractional-order plants, Automatica 61 (2015) 9-17.

[23] H. Liu, Y. Pan, S. Li, Y. Chen, Adaptive fuzzy backstepping control of fractional-order nonlinear systems, IEEE Trans. Syst. Man Cybern. Syst. PP (99) (2016) 1-9.

[24] J. Qiu, H. Gao, S.X. Ding, Recent advances on fuzzy-model-based nonlinear networked control systems: a survey, IEEE Trans. Ind. Electron. 63 (2) (2016) 1207-1217.

[25] H. Pan, W. Sun, H. Gao, X. Jing, Disturbance observer-based adaptive tracking control with actuator saturation and its application, IEEE Trans. Autom. Sci. Eng. 13 (2) (2016) 868-875.

[26] K. Diethelm, The analysis of fractional differential equations, in: An application-Oriented Exposition using Differential Operators of Caputo Type, Lecture Notes in Mathematics 2004, Springer-Verlag, Berlin, 2010.

[27] J.A. Gallegos, et al., On fractional extensions of Barbalat Lemma, Syst. Control Lett. 84 (1) (2015) 7-12.

[28] J.A. Gallegos, M. Duarte-Mermoud, On Lyapunov theory for fractional system, Appl. Math. Comput. 287 (2016) $161-170$

[29] A. Alikhanov, A priori estimates for solutions of boundary value problems for fractional-order equations, Differ. Equ. 46 (5) (2016) 660-666.

[30] K. Narendra, A. Annaswamy, Robust adaptive control in presence of bounded disturbance, IEEE Trans. Autom. Control 31 (1985) 306-315.

[31] J.A. Gallegos, M. Duarte-Mermoud, Boundedness and convergence on fractional order system, J. Comput. Appl. Math. 29 (6) (2016) 815-826.

[32] R. Malti, A note on LP-norms of fractional systems, Autom. (J. IFAC) 49 (9) (2013) 2923-2927.

[33] G. Kavuran, B.B. Alagoz, A. Ates, C. Yeroglu, Implementation of model reference adaptive controller with fractional order adjustment rules for coaxial rotor control test system, Balkan J. Electr. Comput. Eng. 4 (2) (2016).

[34] N.D. Cong, T.S. Doan, H.T. Tuan, Asymptotic stability of linear fractional systems with constant coefficients and small time dependent perturbations, Vietnam J. Math. (2018) 1-16.

[35] J.A. Gallegos, M. Duarte-Mermoud, Robustness and convergence of fractional systems and their applications to adaptive systems, Fract. Calc. Appl. Anal. 20 (4) (2017) 895-913.

[36] N. Aguila-Camacho, M.A. Duarte-Mermoud, J.A. Gallegos, Lyapunov functions for fractional order systems, Commun. Nonlinear Sci. Numer. Simul. 19 (2014) 2951-2957. 